\title{
Finite Element Modeling of Conical Acoustic Emission Sensors and Corresponding Experiments
}

\author{
MARKUS G. R. SAUSE ${ }^{1}$, MARVIN A. HAMSTAD ${ }^{2}$ and SIEGFRIED HORN ${ }^{1}$ \\ 'University of Augsburg, Institute for Physics, Experimental Physics II, D-86135 Augsburg \\ ${ }^{2}$ University of Denver, Department of Mechanical and Materials Engineering, Denver, CO, 80208
}

\begin{abstract}
Mass-backed piezoelectric conical sensor elements are investigated by modeling and corresponding experiments for their response to pencil lead breaks on an aluminum plate. For the experiment and modeling investigation, the plate is chosen large enough to avoid interference of the detected signal by edge reflections within a time frame of $150 \mu \mathrm{s}$. Signals from conical elements with varying cone angle are investigated. For simulation of the sensor signals an approach using multi-scale finite element modeling with coupled partial differential equations is presented. The simulation approach takes into account the signal excitation by pencil lead fracture, formation of Lamb waves, signal propagation and the details of the detection process. This process includes piezoelectric conversion and the influences of the complex impedance of the attached cables and circuitry. Experimental signals and simulated signals are compared as a function of the tip diameter of conical sensor elements. Using the presented method the absolute sensor response can be predicted for arbitrary propagation media and geometries like plates or rods, as well as for alternate sensor geometries.
\end{abstract}

Keywords: acoustic emission, coupled partial differential equation, finite element modeling, conical sensor

\section{Introduction}

In solid materials, rapid transient displacements like formation of cracks, dislocations, corrosion or stick-slip friction can cause small acoustic waves in the ultrasonic range. This phenomenon is known as acoustic emission (AE) and is a valuable tool to investigate failure of engineering materials [1-3]. In order to detect these transient acoustic waves, various detection methods were investigated in the past. The most common type of measurement uses the sensitivity of piezoelectric materials to detect the wave amplitudes down to $2.5 \times 10^{-14} \mathrm{~m}[4,5]$. Based on their frequency response those sensors can be categorized into resonant sensors having one major frequency response, multi-resonant sensors with two or more resonant frequencies and broadband sensors. Typically, the latter ones show relatively flat response within a frequency range that is of interest for $A E$ investigations. These sensors have achieved much attention within the last decades [6-9] and are still being optimized [10]. In addition, various other detection methods were proposed. While mostly capacitance sensors were used in the past decades for broadband applications [11, 
12], recently other methods using fibre-bragg gratings (resonant) [13, 14] or laser interferometers (broadband) have demonstrated their feasibility as approaches to detect $A E[15,16]$. Independent of the principle of detection, it is costly and time consuming to develop broadband and high sensitivity $A E$ sensors by purely experimental methods. A cost and time saving approach is the combination of experiments and a multi-scale finite element modeling approach with coupled partial differential equations that can cover all aspects of the measurement chain.

In recent years finite element modeling was successfully applied to independently model wave propagation in plates [19-23]. The solution for wave propagation with traction free boundary conditions was first described by H. Lamb in 1917 [24], thus these waves are named Lamb-waves. Lamb-waves are guided waves of symmetric and anti-symmetric motion with respect to the mid-plane of the plate. In principle there are unlimited numbers of symmetric and anti-symmetric guided wave modes, but the types of modes faced most often in practical testing are the fundamental symmetric mode $\left(\mathrm{S}_{0}\right)$ and anti-symmetric mode $\left(\mathrm{A}_{0}\right)$.

Moreover, recent attempts demonstrate the feasibility to model response of piezoelectric sensors $[25,26]$. For realistic modeling of sensor signals, aspects of signal propagation and sensor modeling have to be combined as demonstrated in our previous work for a commercial sensor type [18]. This strategy enables a dedicated analysis of the key factors in sensor design, which are responsible for the different transfer functions observed for the various sensor types [17]. Also, a comprehensive modeling strategy of $\mathrm{AE}$ sensors enhances the possibilities to investigate new sensor designs [27-29], or it could give new insight into different sensor concepts to detect AE [29-31].

The purpose of the current work is to develop the specifics of finite element implementation and validate by experiment the finite element method for the case of conical elements of different apertures. In contrast to previous work for sensor modeling [22, 23, 25, 26, 32], the current approach includes signal excitation and signal propagation in a 3-dimensional propagation medium as well as coupled piezoelectric equations to calculate signal voltages based on the deformation of the piezoelectric element. Finally, for the first time, the interaction of the piezoelectric element and the attached circuitry is included by SPICE3 electrical circuit simulation in one software environment.

\section{Modeling of acoustic emission sensors}

While acoustic wave propagation is sufficiently described by solving the constitutive equations of structural mechanics, the interaction between acoustic waves and a piezoelectric sensor requires new approaches using coupled partial differential equations [18, 32]. In particular, the generation of electrical charges due to deformation of the piezoelectric material requires simultaneous solving of the electrical and structural mechanics constitutive equations. Another important aspect in simulation of transfer functions of piezoelectric sensors is the interaction between the sensing material and the attached circuitry. In contrast to conventional open loop simulations of sensor response, additional input impedances of preamplifiers and cables can change the sensor response significantly and have to be taken into account for realistic simulations. 
To include these effects, a finite element modeling approach using the modules "Structural Mechanics" and "AC/DC" of the software COMSOL Multiphysics is applied in this research. In the following, the Einstein summation convention is used.

\subsection{Simulation methodology}

The description of signal excitation and signal propagation within a solid is based on the structural mechanics constitutive equations. Based on the principle of virtual work for an external stimulation the finite element method solves the partial differential equations for equilibrium conditions, expressed in global or local stress and strain tensors $\sigma_{i k}$ and $\varepsilon_{l m}$.

Assuming that the only source of stress $\sigma_{i k}$ is due to strain $\varepsilon_{l m}$ resulting from signal excitation, for linear elastic media with elastic coefficients $C_{i k / m}$, Hook's law is the appropriate constitutive equation.

$$
\sigma_{i k}=C_{i k l m} \cdot \varepsilon_{l m}
$$

For piezoelectric materials, the generation of electrical charges, due to deformation of the crystal lattice, have to be taken into account. Fundamentally, generation of charge density displacements $D_{i}$ is the response of a material due to an external electric field with strength $E_{m}$ linked by the materials electrical permittivity $\chi_{i m}$. The coupled piezoelectric equations use the direct $S_{i k m}$ and inverse $S_{i k m}{ }^{-1}$ piezoelectric constants to connect the structural deformation with the electric response of the material. The formulation of equations (2a) and (2b) in stress-charge form replaces equation (1) as the appropriate constitutive equations for those domains acting as piezoelectric materials.

$$
\begin{aligned}
& \sigma_{i k}=C_{i k l m} \cdot \varepsilon_{l m}-S_{i k m}^{-1} \cdot E_{m} \\
& D_{i}=S_{i k m} \cdot \varepsilon_{l m}+\chi_{i m} \cdot E_{m}
\end{aligned}
$$

The generation of charges is used as a voltage source input for an electrical circuit simulation following the SPICE format developed at University of California, Berkeley. This simulation methodology is based on Kirchhoff's circuit laws and is comprehensively described, i.e. in the original work introducing SPICE3 [33].

\subsection{Simulation setup}

In order to reflect the experimental conditions, a full 3-dimensional approach making use of one xz-symmetry plane introduced at the middle of the sensor (see figure 1-a) was investigated. As the propagation medium, an AIMg3 plate with dimensions of $0.75 \mathrm{~m} \times 0.5 \mathrm{~m}$ and thickness $3.0 \mathrm{~mm}$ was chosen. The geometry of the conical PZT$5 \mathrm{~A}$ elements and the brass backing mass are the same as the experimental values. The piezoelectric elements are truncated cones with a base diameter of $6.5 \mathrm{~mm}$, length of $2.5 \mathrm{~mm}$ and various tip diameters between $0.25 \mathrm{~mm}$ and $4.0 \mathrm{~mm}$. The elastic and piezoelectric properties of the brass backing mass, the PZT-5A conical elements and the aluminium plate are given in table 1 . For simulation of pencil lead breaks (PLB), a point loading is applied to one node of one mesh element located at 
$0.25 \mathrm{~m}$ from the sensor position on the top surface of the plate. As source function, $\mathrm{a}$ linear ramp function following [19] is used.

$$
F(t)= \begin{cases}F_{\text {max }} \cdot\left(t / t_{e}\right) & t \leq t_{e} \\ F_{\max } & t>t_{e}\end{cases}
$$

Within equation (3) $t_{e}$ refers to the excitation time and $F_{\max }$ refers to the maximum out-of-plane force. The excitation time is based on typical values found in literature [34-36]. An evaluation of values between $0.3 \mu \mathrm{s}$ and $2.5 \mu \mathrm{s}$ yielded best comparability with the experimental signals for $1 \mu \mathrm{s}$, which was also confirmed by a previous investigation [37]. The maximum force was estimated as $3 \mathrm{~N}$ by loading until failure the angled (similar to the angle normally used for pencil lead breaks) pencil with the lead extended in a test machine with a sensitive load cell and is found to be in good agreement with experimental data by Scruby et al. [35].

(a)

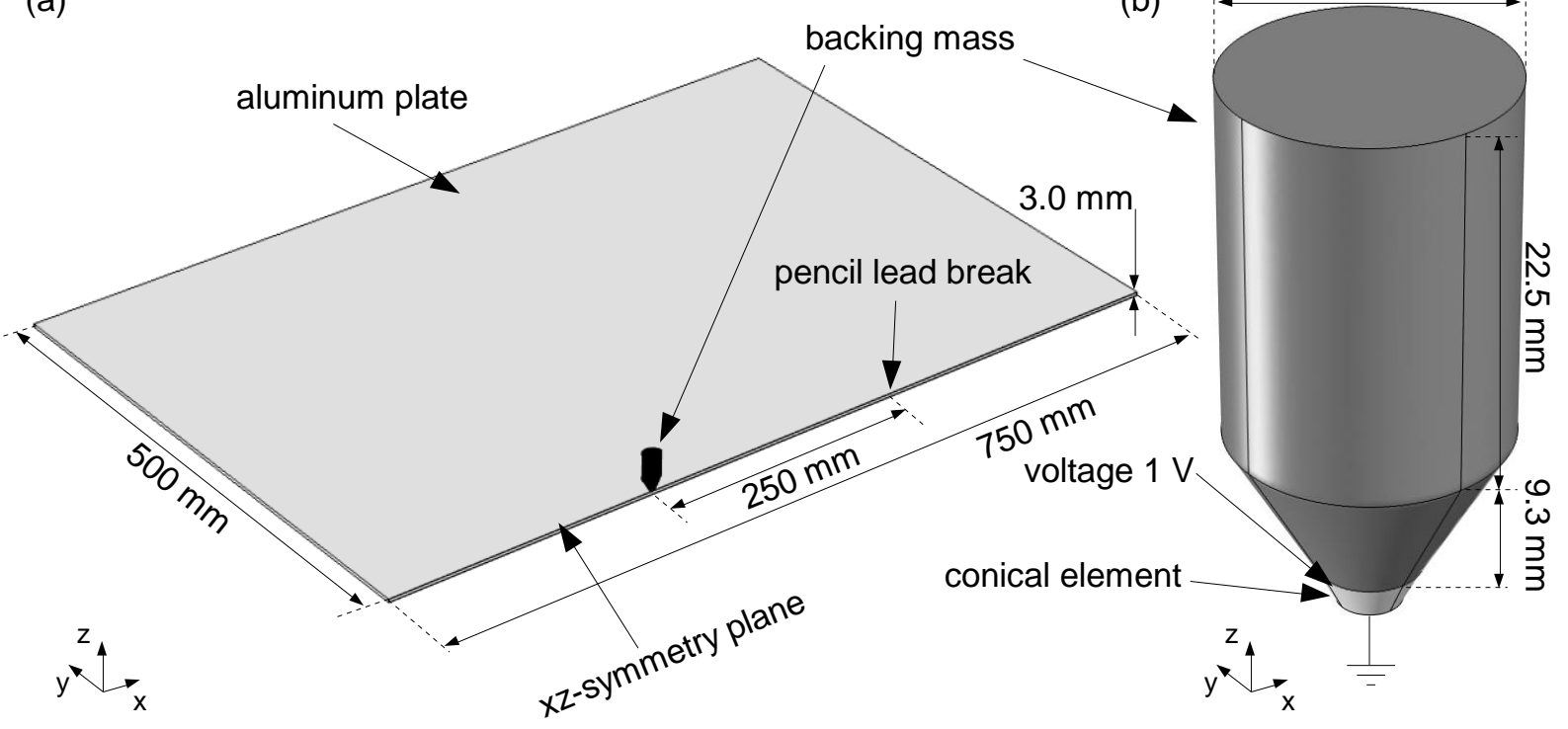

Fig. 1: 3-dimensional model setup of experimental configuration(a) and details of 3-dimensional geometry of sensor for simulation of electric properties (b).

The weight of the sensor was modeled by a $-0.6 \mathrm{~N}$ force applied on top of the backing mass acting in negative z-direction.

For the sensor element, an approach using Rayleigh damping with $\alpha=200000 \mathrm{~s}^{-1}$ and $\beta=1 \times 10^{-8} \mathrm{~s}$ was chosen following the approach of Cervena et al. [25]. As structural boundary conditions, all geometries were connected to form a union. For the boundary conditions of the piezoelectric conversion, the bottom part of the conical sensor element is attached to ground and a terminal circuit boundary condition is used at the top part. The simulated voltage is fed into the circuit simulation shown in figure 2. The attached circuit consists of a cascade of resistor and capacitor elements. The first cascade simulates the impedance of the BNC cable used for electrical connection between preamplifier and sensor. The second cascade is used to model the input impedance and capacitance of the preamplifier. The values for input impedance and capacitance found in figure 2 are chosen based on the manufacturers' datasheets. Finally a $7^{\text {th }}$ order Butterworth high-pass filter at $10 \mathrm{kHz}$ is applied to take into account the systems built-in filter system. 


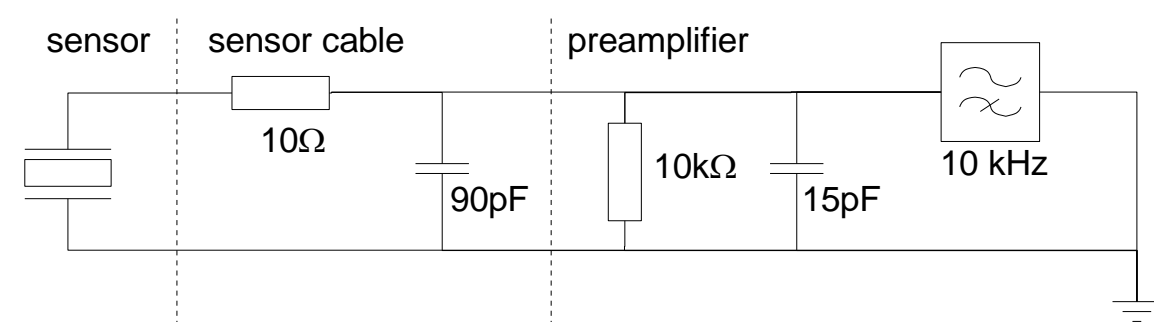

Fig. 2: Drawing of electrical circuit used for simulation of connection cable and preamplifier.

The sensor element and the backing mass were meshed with tetrahedral elements with a maximum size $0.5 \mathrm{~mm}$. The aluminum plate was meshed with increasing mesh size in the $y$-direction at a growth rate of 1.2. The minimum mesh size was chosen at the xz-symmetry plane to be $1.0 \mathrm{~mm}$. To better resolve the geometry of round structures, for the resolution of curvature a factor of 0.6 was used. This setting determines the maximum edge element length, which is given by 0.6 times the curvature radius. All mesh elements are of quadratic geometry shape order. As temporal resolution, a step of $100 \mathrm{~ns}$ was chosen to calculate the first $140 \mu \mathrm{s}$ after signal excitation by simulated PLB.

Convergence of the numerical solution was investigated by calculation of reference cases with higher mesh resolution and higher temporal accuracy. Comparison between signals of the reference case and the calculated signals is based on the coherence level of the signal within the frequency range of interest between $1 \mathrm{kHz}$ and $2 \mathrm{MHz}$. As reference case for spatial mesh resolution a minimum size of $0.4 \mathrm{~mm}$ was found to have a coherence level $\geq 0.99801$, whereas the curvature reference case with curvature factor of 0.1 had a coherence level $\geq 0.99982$. As temporal resolution reference case a 5 ns step size was chosen, which had a coherence level $\geq 0.99996$ to the signals calculated with 100 ns step size. Hence the used settings are suitable to obtain converged results in the frequency range of the experimental signals.

\begin{tabular}{|c|c|c|c|}
\hline Property & AlMg3 & Brass & PZT-5A \\
\hline Density $\rho\left[\mathrm{kg} / \mathrm{m}^{3}\right]$ & 2660 & 8530 & 7750 \\
\hline Elastic Modulus [GPa] & 70.0 & 113.4 & $\begin{array}{l}\mathrm{C}_{11}=\mathrm{C}_{22}=120.3 \\
\mathrm{C}_{12}=75.2 \\
\mathrm{C}_{13}=\mathrm{C}_{23}=75.1 \\
\mathrm{C}_{33}=110.9 \\
\mathrm{C}_{44}=\mathrm{C}_{55}=21.1 \\
\mathrm{C}_{66}=22.6\end{array}$ \\
\hline Poisson ratio & 0.33 & 0.33 & - \\
\hline Piezoelectric constants $S_{i k m}\left[\mathrm{C} / \mathrm{m}^{2}\right]$ & - & - & $\begin{array}{l}S_{31}=S_{32}=-5.4 \\
S_{33}=15.8 \\
S_{24}=S_{15}=12.3\end{array}$ \\
\hline Electrical permittivity $\chi_{\text {im }}$ & - & - & $\begin{array}{l}\chi_{11}=919.1 \\
\chi_{22}=919.1 \\
\chi_{33}=826.6\end{array}$ \\
\hline
\end{tabular}

Tab.1: Elastic and piezoelectric properties of the materials used in the simulation. Subscript indices follow the conventions for piezoelectric materials.

To characterize the sensors electric properties simulations of the impedance and capacitance of the sensor as a function of frequency were performed. Here the full 3dimensional sensor model shown in figure 1-b was used. For the boundary conditions, the bottom part of the conical sensor element was grounded and a 
voltage of $1 \mathrm{~V}$ was applied at the top part with fixed constraint for the structural boundary condition. The resolution of the tetrahedral mesh elements had a maximum size of $0.5 \mathrm{~mm}$. The model was solved as a function of frequency with a step of $10 \mathrm{kHz}$ between $20 \mathrm{kHz}$ and $2 \mathrm{MHz}$.

\section{Experimental}

For comparison with the simulation results experimental signals obtained from PLBs on a $2 \mathrm{~m} \times 1 \mathrm{~m}$ wide and $3 \mathrm{~mm}$ thick AIMg3 aluminum plate were used. Similar to the modeled size of $0.75 \mathrm{~m} \times 1 \mathrm{~m}$, these dimensions are sufficient to exclude reflections from the plate edges within the first $150 \mu$ s after signal excitation, since the conical sensor element with its brass backing are placed at the center of the aluminum plate. For precise positioning and as a stabilizing element (since the brass backing mass balances on top of the conical element), a PMMA tube was used, which allows sliding of the backing mass within. As couplant between brass, conical element and the aluminum plate viscous silicone grease (Apiezon $A$ ) is used. The electrical signal connection was made by a twisted and shielded BNC cable attached with the wire at the top of the brass backing mass and with the ground to the aluminum plate. Ten PLBs with $3.5 \mathrm{~mm}$ length, hardness $2 \mathrm{H}$ and diameter $0.5 \mathrm{~mm}$ were applied at $250 \mathrm{~mm}$ from the midpoint of the sensor. The signals were recorded by a commercial $\mathrm{AE}$ preamplifier and a data acquisition card with 16 bit resolution (model PCl-2). For all signals a bandpass range from $10 \mathrm{kHz}$ to $2 \mathrm{MHz}$ at $40 \mathrm{~dB}$ preamplification and $10 \mathrm{MSP} / \mathrm{s}$ sampling rate was used. To provide a stable trigger, a commercial $\mathrm{AE}$ sensor was placed close to the PLB position to act as guard sensor for the conical sensor under investigation.

\section{Results and Discussion}

In the following, experimental signals and those of the modeling approach are compared to each other. Based on this comparison the change in signal response for different conical element geometries is discussed using additional simulation results.

In principle, a plate thickness of $3.0 \mathrm{~mm}$ as used in this study allows excitation of $\mathrm{S}_{1}$, $S_{2}, A_{1}$ and $A_{2}$ Lamb wave modes within the frequency range up to $2 \mathrm{MHz}$ in addition to the fundamental modes. However, for out-of-plane sources applied to the surface of a plate (e.g. PLBs), signals were found to show only the fundamental Lamb wave modes with dominating $A_{0}$-mode [38]. This is confirmed by modal analysis of the experimental signals as shown in a Choi-Williams distribution with overlapping dispersion curves (see [39, 40] for details). Clearly the signals are dominated by the A 0 -mode as seen from figure 3-a, while use of a truncated time range reveals the contribution of the $S_{0}$-mode at the beginning of the signal (figure 3-b). In figure 4-a, a comparison of four PLB based experimental $A_{0}$-mode signals detected by a conical element with $1.5 \mathrm{~mm}$ tip diameter is shown. The inset shows details of the $\mathrm{S}_{0}$-mode between $40 \mu \mathrm{s}$ and $77 \mu \mathrm{s}$. Although the basic shape of the signal is reproducible, there is some scatter in magnitude and the details of the signals, as was expected due to the nature of PLBs.

Figure 4-b shows a comparison between a representative experimental signal and the simulation result for a respective conical sensor element. The shape of the $\mathrm{S}_{0}-$ mode as shown in the inset of figure 4-b and of the $A_{0}$-mode shown in figure 4-b is described well by the simulation approaches taking into account the scatter of 
experimental signals as seen in figure 4-a. Discrepancies exist in the magnitude at the beginning of the $\mathrm{S}_{0}$-mode and $\mathrm{A}_{0}$-mode. At these points, the simulated signals have lower amplitudes than the experimental signals.

In both the experiment and modeling, the tip diameter of the conical elements was varied over a significant range with a constant base diameter and length to investigate the influence of the geometry on the detected signals. Figure 5 shows a comparison (with a scale appropriate for the $A_{0}$ mode) of the experimental results (fig. 5-a) and simulation results (fig. 5-b). There are two tendencies observed in both the experimental and simulated signals. First, with increasing tip diameter, the signal amplitude is increasing. The increase in absolute voltage values is described well by the simulations. Similar behavior is found for the So-mode, which is shown in more detail in figure 6-a for experimental signals and figure 6-b for simulation results, respectively. Second, with increasing tip diameter, the $A_{0}$-mode becomes somewhat distorted after $100 \mu \mathrm{s}$.
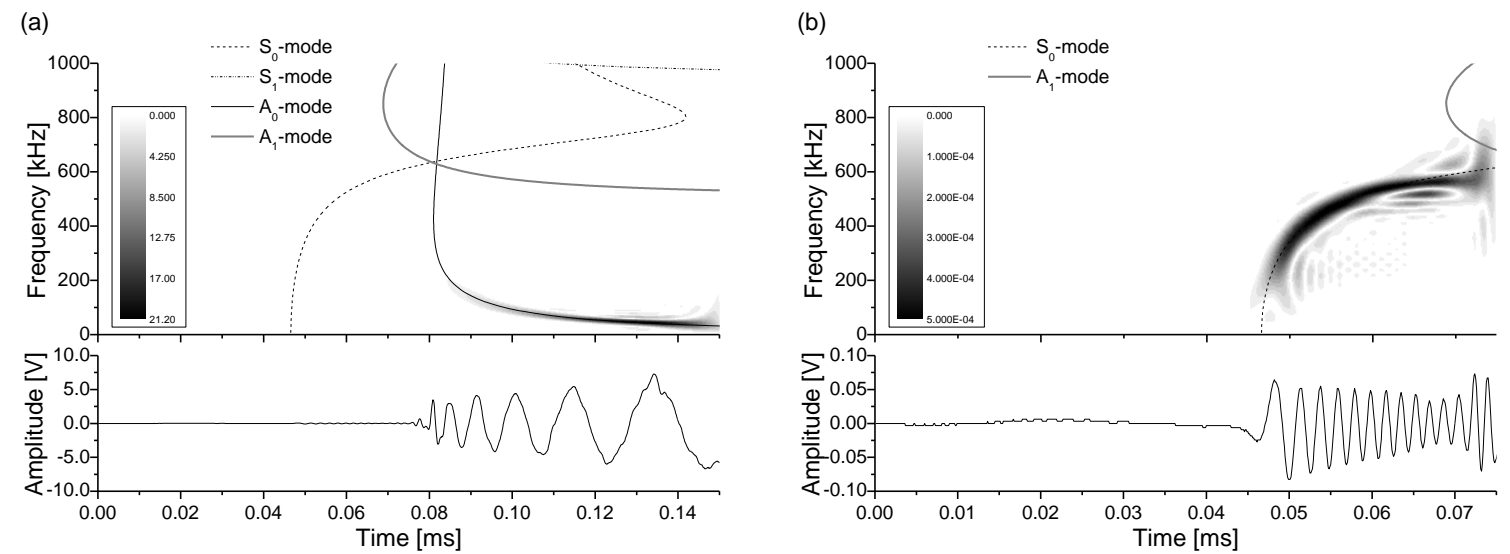

Fig. 3: Choi-Williams distribution of experimental signal in full scale (a) and truncated scale to investigate modal composition of signal (b).
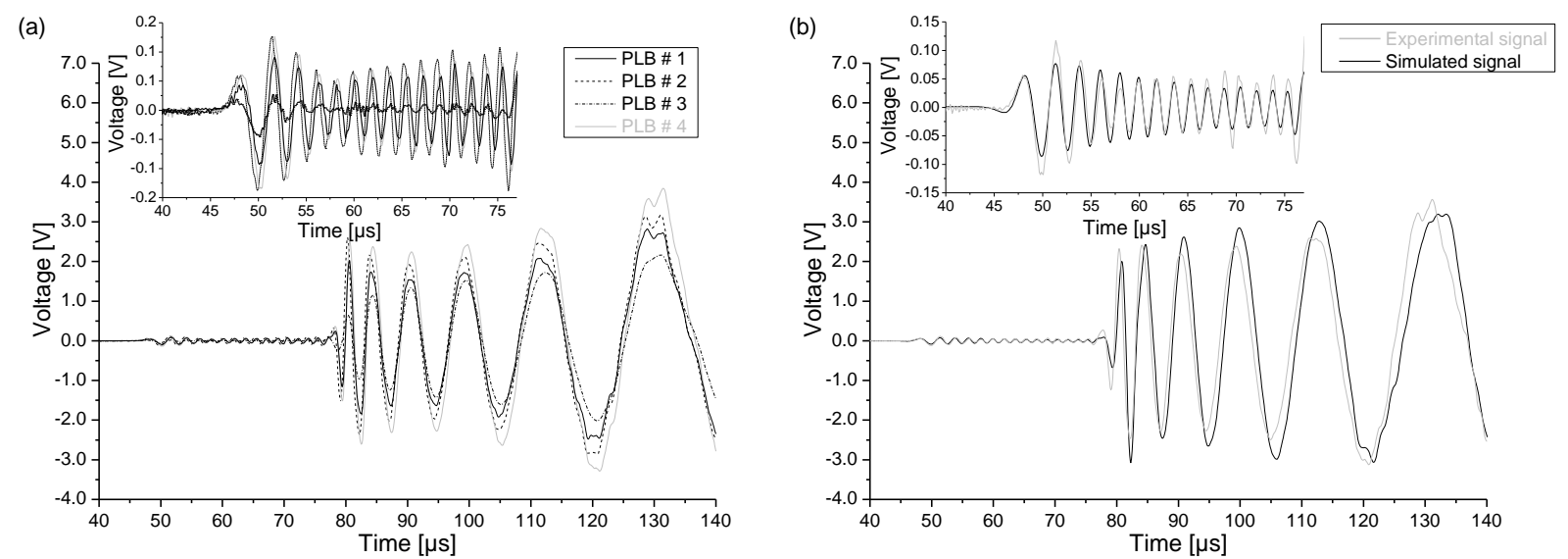

Fig. 4: Scatter of experimental signals of four PLBs (a) and comparison of one experimental signal to simulation results for $1.50 \mathrm{~mm}$ conical element tip diameter (b). Insets show So-mode arrival between $40 \mu \mathrm{s}$ and $77 \mu \mathrm{s}$ after signal excitation. 


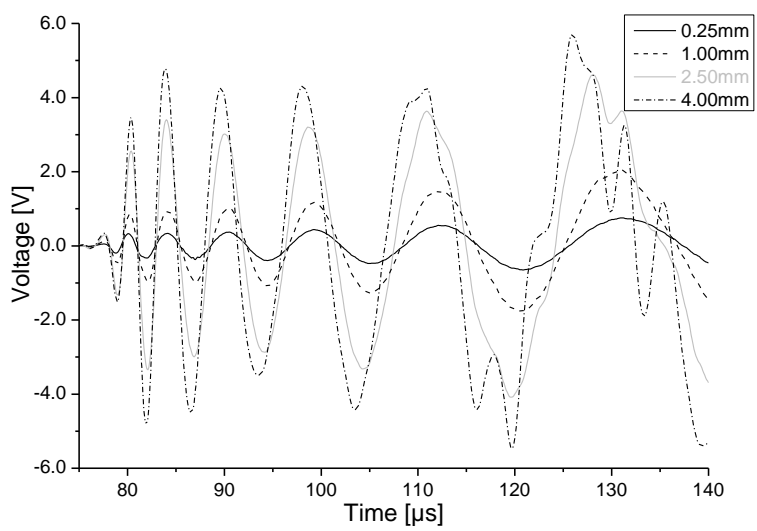

Fig. 5: Comparison between experimental signals element geometries for $A_{0}$ mode region.

(a)

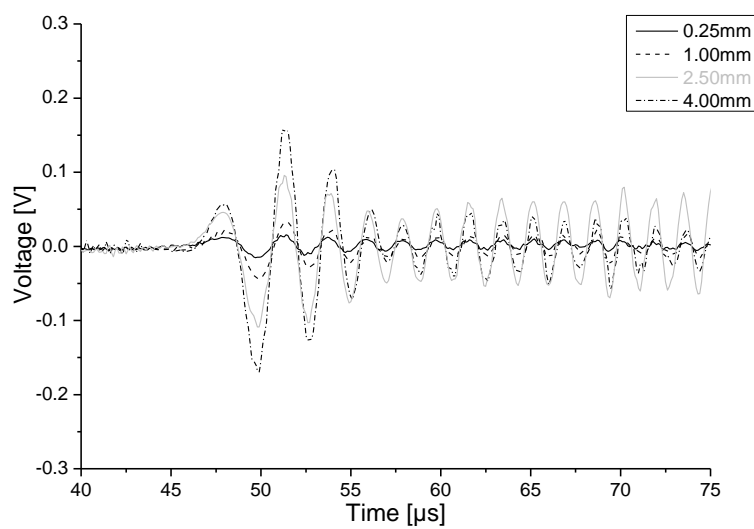

(b)

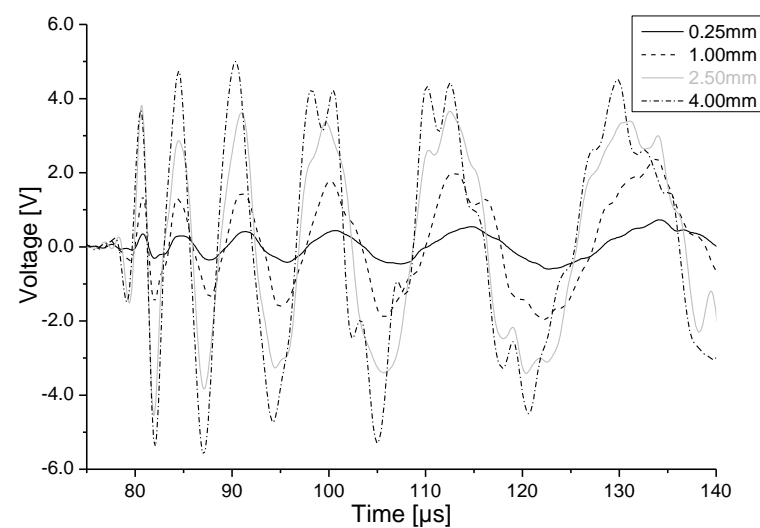

(a) and simulation results (b) for different conical

(b)

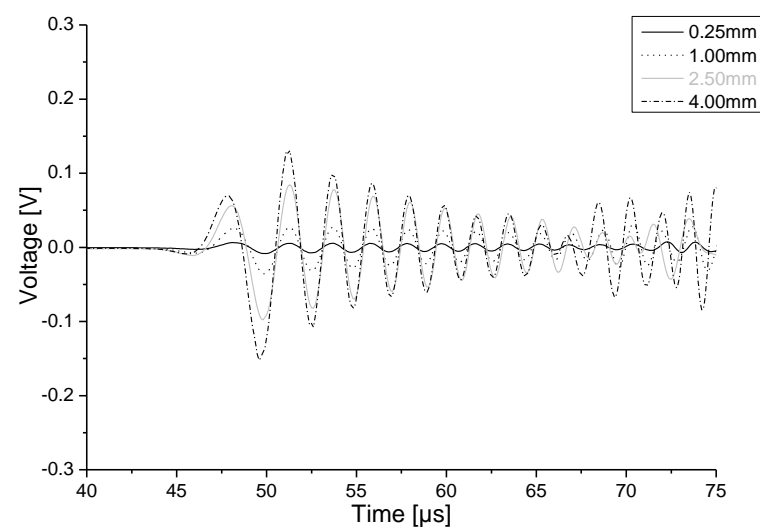

Fig. 6: Comparison between experimental signals (a) and simulation results (b) for different conical element geometries for $\mathrm{S}_{0}$-mode arrival between $40 \mu$ s and $75 \mu$ s after signal excitation.

In order to discuss changes in signal amplitude and signal shape, further simulations of the electrical and structural properties of the various conical element geometries were performed.

The calculated sensor capacitance and impedance for the various conical sensor element geometries are shown as a function of frequency in figures 7-a and 7-b, respectively. There is a clear tendency observed in the calculated capacitance values. For decreasing tip diameter, the curves become flatter, i. e. less dependent on frequency and the capacitance value decreases significantly. Similarly, the sensor impedance increases with decreasing tip diameter, but the characteristic dependency on frequency is retained. Thus all elements show an increase of one to two orders of magnitude in their impedance values when approaching smaller frequencies. Overall, the orders of magnitude calculated for the conical elements agree with values as given by Greenspan et al. [6]. However, based on the current calculated result, it is not suitable to describe piezoelectric elements by one unique value of capacitance or impedance. Instead, those properties should be understood as frequency dependent values. Both properties contribute to the changes in the detected AE signals, since the internal impedance and capacitance of the sensor element form different electrical boundary conditions as a function of frequency. And moreover, the impedance and capacitance are also dependent on the geometry of the sensor element. 
(a)

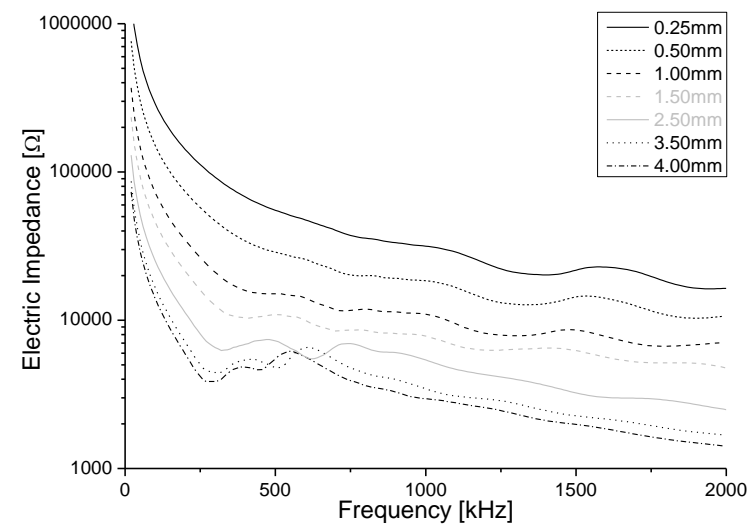

(b)

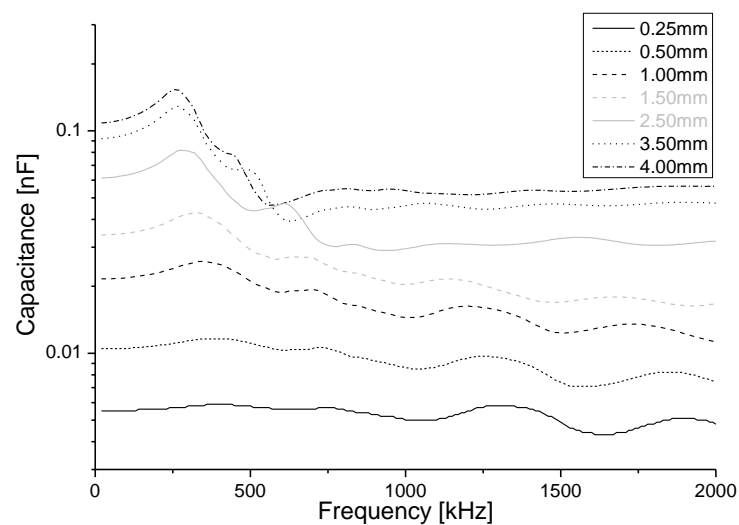

Fig. 7: Calculation results for impedance (a) and capacitance (b) of various conical element geometries as a function of frequency.

To demonstrate the interaction with the attached electrical circuitry, simulations for one conical element with $1.5 \mathrm{~mm}$ tip diameter were performed. First, the capacitance of the cable model was systematically changed between $10 \mathrm{pF}$ and $1000 \mathrm{pF}$, while holding all other circuit parts constant as given in figure 2. The resulting sensor signals of the simulation are shown in figure 8-a. Similarly, the resistance value of the preamplifier was changed from $1 \mathrm{k} \Omega$ to $100 \mathrm{k} \Omega$ (see fig. 8-b), while the cable capacitance was retained. Since the ranges of both cover a broad range of sensor impedance and capacitance (cf. fig. 7-a and fig. 7-b) a strong interaction between the piezoelectric element and the circuitry is found. The change in signal amplitude due to changes in the resistance and capacitance was found to be similar in magnitude to the changes observed from changes in the geometry of the conical elements. For example, an increase in the capacitance of the element has the same effect as a decrease in the circuit capacitance. This is a consequence of the coupled electrical system of piezoelectric material and the attached circuit. Thus, the increase in internal capacitance and the change in impedance of the sensor elements are taken to be a significant cause for the increase in signal amplitude with increasing tip diameter as observed in the experiments and modeled results.
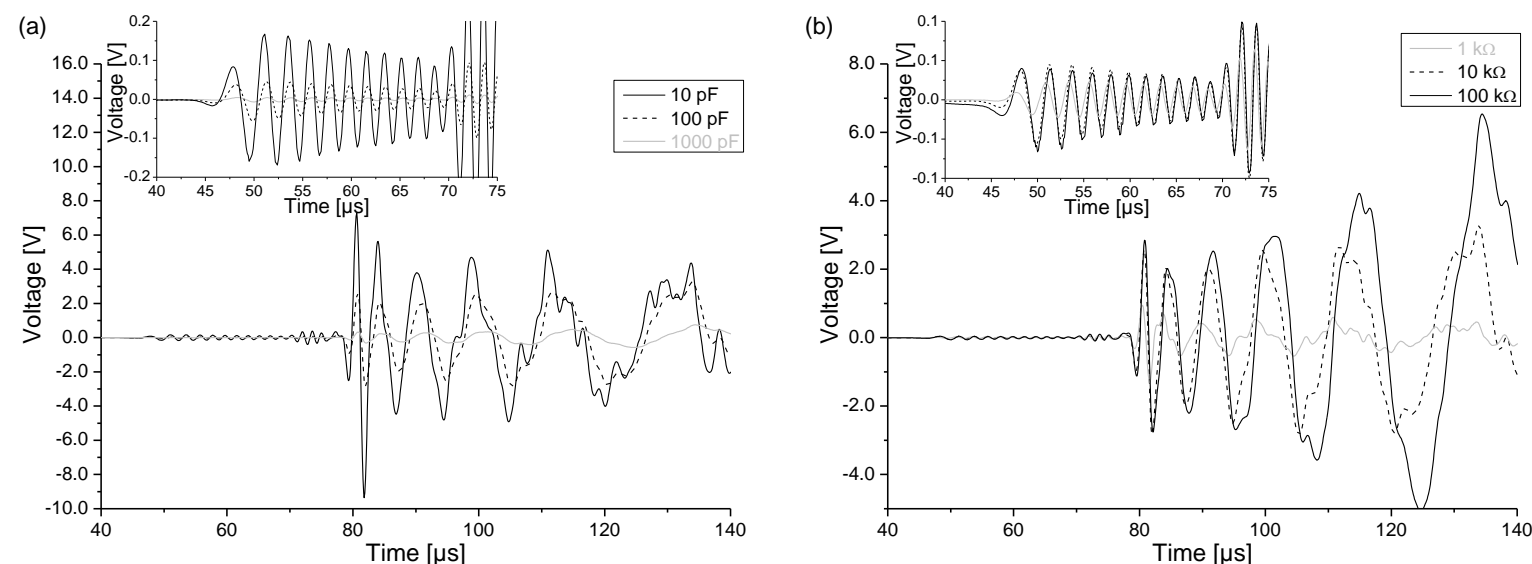

Fig. 8: Variation of cable capacitance for $1.50 \mathrm{~mm}$ conical element diameter (a) and preamplifier impedance (b). Insets show So-mode arrival between $40 \mu$ s and $75 \mu$ s after signal excitation.

In addition to the above, other effects are likely to contribute to the change in shape as well. As discussed previously $[6,10,23,18]$ one major influence on the signal shape is the aperture effect due to the wavelengths of the propagating wave. This influence is pointed out in figure 9-a. A comparison of a simulated signal without presence of the sensor reveals the ideal shape of the Lamb wave in the aluminum 
plate used. An overlay with the normalized simulated sensor voltage signal for $0.25 \mathrm{~mm}$ and $4.00 \mathrm{~mm}$ tip diameters demonstrates the characteristic differences in the signal shape. The out-of-plane signal is in phase with the sensor signals at the beginning of the $A_{0}$-mode, but increasingly deviates at times larger than about $80 \mu \mathrm{s}$. This is caused by the intrinsic phase shift of the piezoelectric conversion and the attached circuit. Despite the phase shift, a better match to the simulated out-of-plane displacement is found for the $0.25 \mathrm{~mm}$ tip diameter. Since this diameter approaches a point contact, the effects of aperture become more negligible. In comparison the $4.00 \mathrm{~mm}$ tip diameter suffers more from an aperture effect and thus shows a signal with more differences compared to the original Lamb wave. Thus, the aperture effect is expected to be responsible for significant changes in the detected signal shapes for the different conical element geometries, but it also contributes to the change in the detected signal amplitudes.
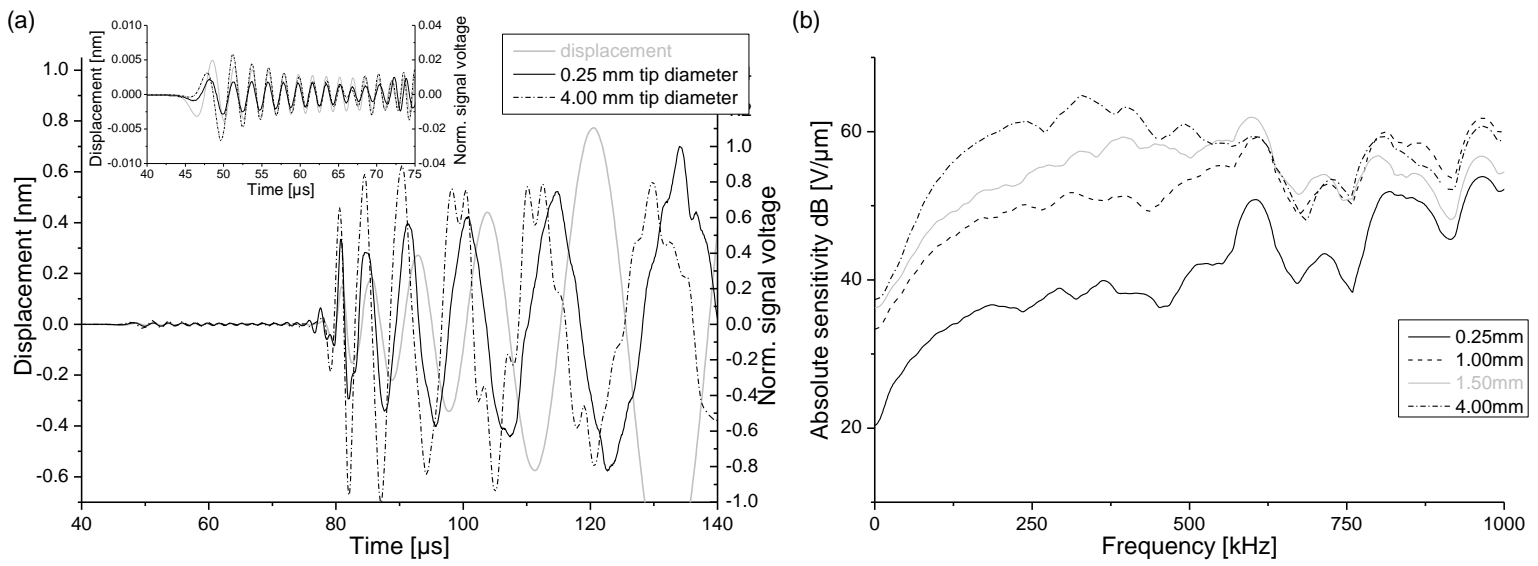

Fig. 9: Comparison between out-of-plane displacement at position of sensor midpoint without presence of sensor model and simulated signal voltage for conical elements with $0.25 \mathrm{~mm}$ and $4.00 \mathrm{~mm}$ tip diameter (a). Absolute sensitivity curves for various conical element geometries as a function of frequency (b).

Using the simulated ideal out-of-plane displacement signal it is possible to discuss the influence of the conical element geometries based on the sensors transfer functions. The calculated absolute sensitivity in $\mathrm{dB}$ with reference to $1 \mathrm{~V} / \mu \mathrm{m}$ are shown in figure 9-b for four of the conical element geometries investigated. Similar to the change in signal voltages seen in figures 5 and 6 , there is a significant change in the calculated absolute sensitivity of the various conical element geometries. For the conical element tip diameters between $0.25 \mathrm{~mm}$ and $1.00 \mathrm{~mm}$ the absolute sensitivity increases steadily, while the frequency response is almost retained within the shown range up to $1 \mathrm{MHz}$. For the conical elements tip diameters above $1.00 \mathrm{~mm}$, the sensitivity still increases below $500 \mathrm{kHz}$, but the frequency response above $500 \mathrm{kHz}$ suffers from higher attenuation. Generally, at frequencies above $500 \mathrm{kHz}$ all curves show stronger distortions, i.e. the curves become less flat.

\section{Conclusion}

It has been demonstrated how finite element modeling strategies can be used to calculate $A E$ sensor signals and $A E$ sensor transfer functions. The simulation method uses a comprehensive approach taking into account in-situ placement of an $A E$ sensor on a propagation medium, piezoelectric conversion and the interaction of the sensor material with an electric circuit. For validation of the simulation method, experiments on an aluminum plate using PLBs as an AE source were conducted. 
The simulated signals obtained for seven different conical element geometries show systematic agreement to the experimental signals. In the experiments, the electrical signal strength increases with increasing diameter, but the shape of the Lamb wave modes gets distorted. Using additional simulations of electrical and structural properties of the sensor, reasons for these changes in the sensor response were investigated.

Based on these simulations it was demonstrated, that one major influence on the shape and amplitude of the detected signals is the attached circuit. It is worth noting, that simulations conducted without attached electrical circuit (open loop) turned out to yield an inverse evolution of signal amplitudes as a function of conical element tip diameter. Thus a simulation neglecting the influence of the attached circuitry cannot explain the behavior seen in the experiment. Moreover, the interaction between the internal capacitance and impedance of the conical sensor element with the attached electronic devices can significantly influence the signal shape.

It was found that the aperture effect has significant impact on the signal shape. This partially explains why conical elements typically show flat frequency response. Since the tip of the conical element is of smaller diameter than most commercial $\mathrm{AE}$ sensors and is smaller than the signal wavelengths, the aperture effect becomes negligible. For the widely used piezoelectric disc or ring elements the influence of the aperture effect was already found to be even more significant $[18,23]$.

In general, the presented approach is feasible for simulation of all piezoelectric sensing elements that focus on detection of acoustic waves. In particular, this refers to the widely used commercial $A E$ sensors, given that the internal structure of the sensor and the materials used are well known.

Therefore, the combination of finite element simulations and experiment has demonstrated that the proposed method can be used to predict sensor transfer functions in arbitrary geometries of propagation media and for arbitrary materials. Ultimately, the presented method can also be used as a tool to conduct numerical studies within the scope of sensor optimization in terms of sensitivity or frequency response. In the current examination, both the modeled and experimental results pointed to a $1.5 \mathrm{~mm}$ tip diameter for conical elements to be the best when considering both highest amplitude response sensitivity and the best flatness of the spectral response.

\section{Literature}

[1] M. A. Hamstad, Thirty Years of Advances and Some Remaining Challenges in the Application of Acoustic Emission to Composite Materials, in: T. Kishi, M. Ohtsu, S. Yuyama (Eds.), Acoustic Emission - Beyond the Millenium, Elsevier, Tokyo, 2000, pp. 77-91.

[2] C. R. Heiple, S.H. Carpenter, Acoustic Emission Produced by Deformation of Metals and Alloys - A Review: Part I, J. Acoustic Emission 6:2 (1987) 177-204.

[3] C. R. Heiple, S.H. Carpenter, Acoustic Emission Produced by Deformation of Metals and Alloys - A Review: Part II, J. Acoustic Emission, 6:2 (1987) 215-237.

[4] E. S. Boltz, C. M. Fortunko, M. A. Hamstad, M. C. Renken, Absolute Sensitivity of air, light and direct-coupled wideband acoustic emission transducers, in: D. O. Thompson, D. E. Chimenti (Eds.) Review of Progress in Quantitative Nondestructive Evaluation 14, Plenum Press, New York, 1995, pp. 967-974.

[5] C. A. Tatro, Design Criteria for Acoustic Emission Experimentation, in Acoustic Emission, ASTM STP 505, American Society for Testing and Materials, Phil. PA, 1972, pp. 84-99.

[6] M. Greenspan, The NBS conical transducer: Analysis, J. Acoust. Soc. Am. 81:1 (1987) 173-183.

[7] M. A. Hamstad, C. M. Fortunko, Development of practical wideband high-fidelity acoustic emission sensors, in: S. Chase (Ed.) Nondestructive Evaluation of Aging Bridges and Highways, SPIE-2456, 1995, pp.281-288. 
[8] M. A. Hamstad, Improved signal-to-noise wideband acoustic/ultrasonic contact displacement sensors for wood and polymers, Wood Fiber Sci. 29:3 (1997) 239-248.

[9] M. R. Gorman, Plate Wave Acoustic Emission, J. Acoust. Soc. Am. 90:1 (1991) 358-364.

[10] G. McLaskey, S. Glaser, Hertzian impact: Experimental study of the force pulse and resulting stress waves, J. Acoust. Soc. Am. 128 (2010) 1087-1096.

[11] F. R. Breckenridge, C. E. Tschiegg, M. Greenspan, Acoustic emission: some applications of Lamb's problem, J. Acoust. Soc. Am. 57 (1975) 626-631.

[12] C. B. Scruby, H. N. G. Wadley, A calibrated capacitance transducer for the detection of acoustic emission, J. Phys. D. Appl. Phys 11 (1978) 1487-1494.

[13] I. Read, P. Foote, S. Murray, Optical fibre acoustic emission sensor for damage detection in carbon fibre composite structures, Meas Sci. Technol. 13 (2002) N5-N9.

[14] G. Wild, S. Hinckley, Fiber Bragg Grating Sensors for Acoustic Emission and Transmission Detection Applied to Robotic NDE in Structural Health Monitoring, in: Sensors Applications Symposium, IEEE, 2007, pp.1-6.

[15] S. Weissner, F. E. Talke, Load/unload measurements using laser doppler vibrometry and acoustic emission, Tribol. Int. 33:5-6 (2000) 367-372.

[16] M. Watanabe, M. Enoki, T. Kishi, Fracture behavior of ceramic coatings during thermal cycling evaluated by acoustic emission method using laser interferometers, Mat. Sci. Eng. A 359:1-2 (2003) 368-374.

[17] K. Ono, H. Cho, T. Matsuo, Transfer functions of acoustic emission sensors, J. Acoustic Emission 26 (2008) 72-90.

[18] M. G. R. Sause, Identification of failure mechanisms in hybrid materials utilizing pattern recognition techniques applied to acoustic emission analysis, PhD-dissertation, mbv-Verlag, Berlin, 2010.

[19] J. Gary, M. A. Hamstad, On the Far-field Structure of Waves Generated by a Pencil Lead Break on a Thin Plate, J. Acoustic Emission 12:3-4 (1994) 157-170.

[20] W. H. Prosser, M. A. Hamstad, J. Gary, A. O. Gallagher, Finite Element and Plate Theory Modeling of Acoustic Emission Waveforms, J. Nondest. Eval. 18:3 (1999) 83-90.

[21] M. Castaings, C. Bacon, B. Hosten, M. V. Predoi, Finite element predictions for the dynamic response of thermo-viscoelastic material structures, J. Acoust. Soc. Am. 115:3 (2004) 1125-1133.

[22] D. W. Greve, J. J. Neumann, J. H. Nieuwenhuis, I. J. Oppenheim, N. L. Tyson, Use of Lamb waves to monitor plates: experiments and simulations, in: Proceedings of the Society of Photo-Optical Instrumentation Engineers, SPIE-5765, 2005, pp. 281-292

[23] M. G. R. Sause, S. Horn, Simulation of acoustic emission in planar carbon fiber reinforced plastic specimens, J. Nondest. Eval. 29:2 (2010) 123-142.

[24] H. Lamb, On Waves in an Elastic Plate, P. Roy. Soc. Lond. A Mat. 93 (1917) 114-128.

[25] O. Cervena, P. Hora, Analysis of the conical piezoelectric acoustic emission transducer, Applied and Computational Mechanics 2 (2008) 13-24.

[26] Y.-H. Huang, C.-C. Ma, Forced vibration analysis of piezoelectric quartz plates in resonance, Sensors Actuat. A-Phys. 149:2 (2009) 320-330.

[27] G.-H. Feng, M.-Y. Tsai, Acoustic emission sensor with structure-enhanced sensing mechanism based on micro-embossed piezoelectric polymer, Sensors Actuat. A-Phys. 162:1 (2010) 100-106.

[28] S. W. Or, H. L. W. Chan, C. L. Choy, P(VDF-TrFE) copolymer acoustic emission sensors, Sensors Actuat. A-Phys. 80:3 (2000) 237-241.

[29] M. Barbezat, A. J. Brunner, P. Flueler, C. Huber, X. Kornmann, Acoustic emission sensor properties of active fibre composite elements compared with commercial acoustic emission sensors, Sensors Actuat. A-Phys. 114:1 (2004) 13-20.

[30] P. Marin-Franch, T. Martin, D. L. Tunnicliffe, D. K. Das-Gupta, PTCa/PEKK piezo-composites for acoustic emission detection, Sensors Actuat. A-Phys. 99:3 (2002) 236-243.

[31] S.X. Zheng, R. McBride, J.S. Barton, J.D.C. Jones, K.F. Hale, B.E. Jones, Intrinsic optical fibre sensor for monitoring acoustic emission, Sensors Actuat. A-Phys. 31:1-3 (1992) 110-114.

[32] J. H. Nieuwenhuis, J. Neumann, D. W. Greve, I. J. Oppenheim, Generation and detection of guided waves using PZT wafer transducers, IEEE T. Ultrason. Ferr. 52 (2005) 2103-2111.

[33] T. L. Quarles, Analysis of Performance and Convergence Issues for Circuit Simulation, Ph. D. dissertation, Electr. Electron. Comp. Sci. Dept., Univ. Calif. Berkeley, Elec. Res. Lab., Apr. 1989.

[34] F. R. Breckenridge, T. M. Proctor, N. N. Hsu, S. E. Fick, D. G. Eitzen, Transient sources for acoustic emission work, in: K. Yamagushi, H. Takakashi, and H. Niitsuma (Eds.) Progress in Acoustic Emission V, Tokio, 1990, pp. 20-37.

[35] C. B. Scruby, J. C. Collingwood, H. N. G. Wadley, A new technique for the measurement of acoustic emission transients and their relationship to crack propagation, J. Phys. D. Appl. Phys 11 (1978) 2359-2369. 
[36] R. A. Kline, The Influence of Attenuation on Acoustic Emission Measurements, IEEE 1984 Ultrasonics Symposium (1984) 930-932.

[37] M. G. R. Sause, Investigation of Pencil-Lead Breaks as Acoustic Emission Sources, J. Acoustic Emission 29 (2011) $184-196$.

[38] M. A. Hamstad, On Lamb Modes as a Function of Acoustic Emission Source Rise Time, J. Acoustic Emission 28 (2010) $41-58$.

[39] H.-I. Choi, W. Williams. Improved Time-Frequency Representation of Multicomponent Signals Using Exponential Kernels, IEEE T. Acoust., Speech 37:6 (1989) 862 - 872.

[40] M. A. Hamstad. Comparison of Wavelet Transform and Choi-Williams Distribution to Determine Group Velocities for Different Acoustic Emission Sensors, J. Acoustic Emission 26 (2008) 40 - 59.

Vitae:

Markus G. R. Sause received his Ph.D. degree in physics from the University of Augsburg, Germany in 2010. His field of research includes acoustic emission of key engineering materials, in particular fiber reinforced composites, pattern recognition techniques and multi-scale finite element simulations.

Marvin A. Hamstad received his Ph.D. degree from the University of California, Berkeley, California, USA in the field of Applied Mechanics. He spent 13 years at the Lawrence Livermore National Laboratory doing research in multiple areas of acoustic emission technology and also composite materials and structures. He followed that spending 26 years as professor at the University of Denver doing research in the same fields. He currently is a professor emeritus. He has also been associated with the National Institute of Standards and Technology in Boulder, CO for 20 years working in the AE field.

Siegfried Horn received his Ph.D. degree from the Technical University Darmstadt. He spent five years at Polytechnic Institute of New York University doing research in spectroscopy. Since 1992 he is professor at the University of Augsburg, Germany doing research in the field of materials science, in particular on surfaces and interfaces. 